\title{
BMJ Open Clinical characteristics and lipid lowering treatment of patients initiated on proprotein convertase subtilisin- kexin type 9 inhibitors: a nationwide cohort study
}

Jakob Solgaard Jensen, ${ }^{1}$ Peter Ejvin Weeke, ${ }^{2}$ Lia Evi Bang, ${ }^{1}$ Dan Eik Høfsten, ${ }^{1}$ Maria Sejersten Ripa, ${ }^{3,4}$ Anne-Marie Schjerning, ${ }^{3}$ Juliane Elizabeth Theilade, ${ }^{3}$ Lars Valeur Køber, ${ }^{1}$ Gunnar Hilmar Gislason, ${ }^{3}$ Jannik Pallisgaard ${ }^{3}$

To cite: Jensen JS, Weeke PE, Bang LE, et al. Clinical characteristics and lipid lowering treatment of patients initiated on proprotein convertase subtilisin-kexin type 9 inhibitors: a nationwide cohort study. BMJ Open 2019;9:e022702. doi:10.1136/ bmjopen-2018-022702

- Prepublication history and additional material for this paper are available online. To view these files, please visit the journal online (http://dx.doi org/10.1136/bmjopen-2018022702).

Received 29 April 2018 Revised 11 September 2018 Accepted 3 January 2019
D) Check for updates

(c) Author(s) (or their employer(s)) 2019. Re-use permitted under CC BY-NC. No commercial re-use. See rights and permissions. Published by BMJ.

For numbered affiliations see end of article.

\section{Correspondence to} Jakob Solgaard Jensen; jakob.solgaard.jensen@regionh. $\mathrm{dk}$

\section{ABSTRACT}

Objectives Given the novelty of proprotein convertase subtilisin-kexin type 9 inhibitors (PCSK9i), little is known regarding overall implementation or clinical characteristics among patients who initiate treatment. We aimed to assess the total number of patients initiated on PCSK9i along with a description of the clinical characteristics and lipid lowering treatment (LLT) of such patients.

Setting A register-based descriptive cohort study of patients receiving a PCSK9i in the time period from 01 January 2016 to 31 March 2017 using a cross linkage between three nationwide Danish registers. Information regarding PCSK9i prescriptions, patient demographics, concurrent pharmacotherapy, comorbidities and previous coronary procedures was identified.

Results Overall, 137 patients initiated treatment with PCSK9i in the study period from 11 in the first quarter of 2016 to 40 in the first quarter of 2017. The majority had a history of ischaemic heart disease (IHD) $(67.9 \%)$ with ischaemic stroke and diabetes mellitus being present in $7.3 \%$ and $16.8 \%$ of patients, respectively. All patients initiated on PCSK9i had been previously prescribed statin treatment with atorvastatin and simvastatin being most frequently prescribed in $53 \%$ and $36 \%$ of patients, respectively. The majority of patients had received both statins and ezetimibe (94.9\%) and approximately half of these patients had also received bile acid sequestrant (45.3\%). Clinical characteristics mainly differed in patients receiving triple LLT compared with patients not receiving triple LLT in the regards of heart failure.

Conclusion Patients treated with PCSK9i were rare, characterised by having IHD and had received various and intensive conventional LLT prior to PCSK9i initiation in agreement with current international guidelines.

\section{INTRODUCTION}

Atherosclerosis-related morbidity and mortality is reaching epidemic proportions worldwide and complications related to ischaemic heart disease (IHD) are the leading cause of death under 65 years of age. ${ }^{1}$
Strengths and limitations of this study

- In the present study, we utilised the Danish national registries to perform a description of the characteristics of patients prescribed with PCSK9i on a national basis. We were able to include an unselected cohort of patients, with a great variety of comorbidities.

- The Danish nationwide registries include data on all residents regardless of participation in the labour market, and therefore is not affected by selection bias caused by including selected age groups, hospitals or health insurance systems.

- Patients with familial hypercholesterolaemia (FH), who are also eligible for PCSK9i treatment, collect their PCSK9i at a specialised hospital unit and due to the design of the present study, we were not able to identify patients with a diagnosis with $\mathrm{FH}$.

- Due to the observational registry design of the study, we were not able to retrieve data regarding biochemical analyses, clinical arguments for initiation of PCSK9i, LLT after initiation of PCSK9i or adverse events with LLT.

- Due to a low diagnostic sensibility, we could not fully discriminate the indications for patients receiving PCSK9i.

Low-density lipoprotein cholesterol (LDL-C) is a well-established and modifiable constituent in the pathogenesis of atherosclerosis and epidemiological studies have established LDL-C as one of the main risk factors for the development of atherosclerotic disease and IHD. $^{1-3}$

Since the late 1980s, statins have been used to lower LDL-C levels and reduce the risk of cardiovascular events and related mortality across the spectrum of primary and secondary prevention. $^{4-6}$

Randomised trials have since targeted even lower LDL-C levels achieved by intensified 
LDL-C lowering treatment (ie, higher dosage, more potent statins or additional ezetimibe) in patients with IHD, which has shown to further reduce the risk of cardiovascular disease (CVD).$^{7-10}$

Novel treatment options to further lower the LDL-C levels are monoclonal antibodies to proprotein convertase subtilisin-kexin type 9 (PCSK9), which have shown lowering of the LDL-C levels by $40 \%-60 \%$ and relative reduction of cardiovascular events by $15 \%$ (CI: 0.79-0.92) when added to background statin treatment. ${ }^{11}$

The European Society of Cardiology (ESC) and The American College of Cardiology (ACC) guidelines ${ }^{12} 13$ have endorsed PCSK9i treatment in patients at high risk and very high risk of future cardiovascular events. Partly due to the high cost of PCSK9i, most countries have established national guidelines and committees to approve a prior authorisation application for medicine subsidy in the individual patient. In Denmark, PCSK9i treatment were approved for medication subsidy in patients in very high risk of future CVD (ie, patients with a history of acute coronary syndrome, acute myocardial infarction, atherosclerotic polyvascular disease or diabetes mellitus with IHD) and in patients in high risk of future CVD (ie, patients with a history of stable angina pectoris or diabetes mellitus with either target organ damage, peripheral atherosclerotic artery disease, transitory cerebral ischaemia or ischaemic cerebral infarction) who despite maximally tolerated lipid lowering treatment (LLT) required further reduction of LDL-C levels. Established cut-off LDL-C values were $3.0 \mathrm{mmol} / \mathrm{L}(115 \mathrm{mg} / \mathrm{dL})$ and $3.5 \mathrm{mmol} / \mathrm{L}(135 \mathrm{mg} / \mathrm{dL})$ in the very high-risk patients and high-risk patients, respectively (online supplementary appendix figure 1). Statin intolerance in these patients would also be approved for subsidy when treatment with at least three types of statin starting in low dosages titrated to maximum tolerated dosage and ezetimibe and bile acid sequestrant had been attempted prior to PCSK9i initiation. The ESC and ACC guidelines further endorse additional clinical criteria (ie, patients with a history of isolated peripheral atherosclerotic artery disease, isolated ischaemic cerebral infarction or diabetes mellitus with marked hypertension or hypercholesterolaemia) and at different LDL-C measurement cut-off values. In these patients, however, there is a lack of treat to target trials and the effects of intensified LDL-C lowering is not with thorough evidence. ${ }^{14}{ }^{15}$ Given the novelty of PCSK9i and the difference in national and international guidelines, we do not know the exact prevalence, the clinical characteristics, concurrent medication or the attempts of double and triple LLT in patients initiated on PCSK9i.

In the present study, we wanted to assess the total number of patients initiated on PCSK9i in the first quarter (Q1) of 2016 to the Q1 of 2017 with a description of the clinical patient characteristics, comorbidities, medication use, concurrent LLT in these patients. Furthermore, we wanted to compare these parameters in patients attempted in triple LLT and patients in single or double LLT.

\section{METHODS}

In this register-based cohort study, information regarding patient demographics, comorbidities, coronary procedures and concurrent medication use was identified using cross linkage between three different nationwide Danish registers. The Civil Registration System holds information on date of birth, sex and survival status. The Danish National Patient Register holds information on every hospital admission in Denmark since 1978, in which each hospitalisation is registered at discharge with one primary diagnosis and, if applicable, one or more secondary diagnoses according to the International Classification of Diseases, the 10th revision (ICD-10) since 1994. The Danish National Patient Register also holds information on surgeries and procedures including percutaneous coronary intervention (PCI) and Coronary Artery Bypass Grafting (CABG). Comprising data on date, quantity, strength, formulation of all prescriptions dispensed from Danish pharmacies has been accurately registered in The Danish Registry of Medicinal Product Statistics since 1995 and coded according to the Anatomical Therapeutic Chemical (ATC) classification system.

All Danish patients with a prescription for PCSK9i (ATC code $\mathrm{C} 10 \mathrm{~A} \times 13$ or $\mathrm{C} 10 \mathrm{~A} \times 14$ ) between 1 January 2016 and 31 March 2017 were included in the study cohort on the day they redeemed their prescription. Since 1 March 2017, PCSK9i were collected at specialised units at Danish hospitals and prescriptions of PCSK9i in the time period from 1 March 2017 to 31 March 2017 were not registered in the present study.

Demographic information on age, sex and vital status was identified using The Civil Registration System. Comorbidities and coronary procedures were identified using The Danish National Patient Register. Information on diabetes mellitus and hypertension was identified using The Danish Registry of Medicinal Product Statistics along with the concurrent medication (online supplementary appendix table 1 ).

\section{Statistics}

Categorical data is presented as counts with percentages. Statistical difference was tested using chi-squared test or Fisher's exact test where appropriate. Continuous variables were reported as medians with IQR. Statistical difference was tested using the Wilcoxon rank-sum test. A $p$ value $<0.05$ was considered significant.

Data management and statistical analyses were conducted using R statistics, V.3.4.3 (R Core Team [2017]. $\mathrm{R}$ : A language and environment for statistical computing. R Foundation for Statistical Computing, Vienna, Austria. URL http://www.R-project.org/).

\section{Ethics}

In Denmark, retrospective register studies do not require approval from the ethics committees. The ethical legislation in Denmark does not permit the reporting of clinical patient characteristics in less than three patients. Data were made available in an 


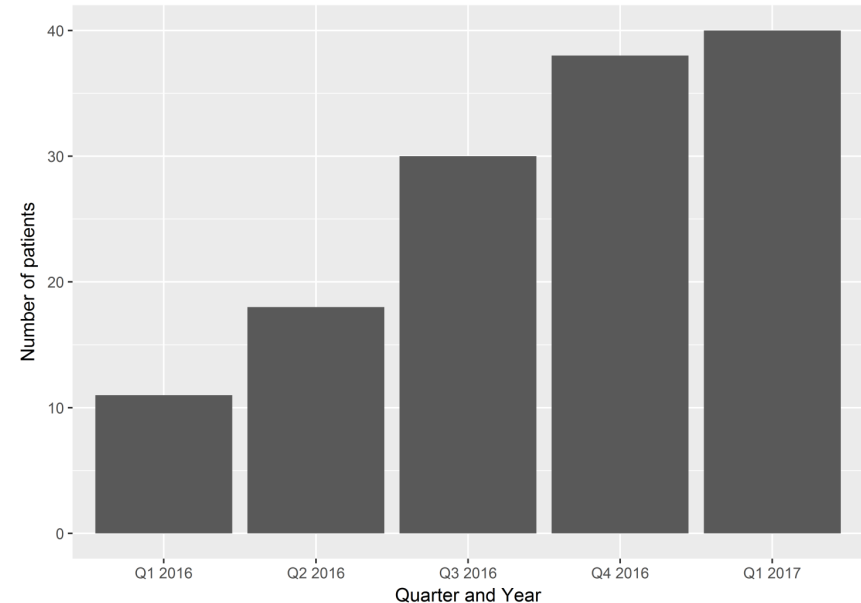

Figure 1 Bar plot illustrating the number of patients initiated with PCSK9 inhibitor treatment by calendar quarter. Q1 2016=01 January 2016-31 March 2016; Q2 2016=01 April 2016-30 June 2016; Q3 2016=01 July 2016-30 September 2016; Q4 2016=01 October 2016-31December 2016; Q1 2017=01 January 2017-31 March 2017.

anonymised format such that specific individuals could not be identified. The Danish Data Protection Agency approved this study (ref. no.: 2007-58-0015/GEH-2014016 I-Suite no.: 02734)

\section{Patient and public involvement}

Patients and public were not involved in this research project.

\section{RESULTS}

The total number of patients treated with PCSK9i during the total study period of 15 months was 137 . The total number of patents initiating PCSK9i increased from 11 in Q1 of 2016 to 40 in Q1 of 2017 (figure 1). The overall median age was 62 (IQR: 55-68) years and 53.3\% were men with no difference in number of men and women initiated (online supplementary appendix figure 2 ). The majority of patients had a history of IHD (68\%) with $51.1 \%$ having received PCI and $24.8 \%$ having received CABG. All patients had filled a prescription of statin $(100 \%)$ prior to initiation of PCSK9i with atorvastatin and simvastatin being most frequently prescribed in $57.7 \%$ and $32.1 \%$ of patients, respectively (table 1 ).

The majority of patients had received statins in dosages of $80 \mathrm{mg}(43.8 \%)$ or $40 \mathrm{mg}(46.7 \%)$. Of the 137 included patients, $94.9 \%$ of patients had filled a prescription of ezetimibe in addition to statin and $43.1 \%$ of patients had filled a prescription of three types of LLT with bile acid sequestrants and ezetimibe in addition to statin. Few patients had filled a prescription of statin alone $(2.9 \%)$ and $50 \%$ of all patients had filled a prescription of either fibrates or nicotinic acid (figure 2 and table 1 ).

Patients in dual LLT were more prone to have heartfailure than patients attempted in triple LLT prior to
Table 1 Clinical characteristics, demographics and medication use of patients initiated on PCSK9 inhibitors

\begin{tabular}{|c|c|}
\hline Parameter & Study cohort \\
\hline Number of patients, $n$ & 137 \\
\hline Age (median (IQR)) & $62(55-68)$ \\
\hline Men, $\mathrm{n}(\%)$ & $73(53.3)$ \\
\hline Ischaemic stroke, n (\%) & $10(7.3)$ \\
\hline Ischaemic heart disease, $\mathrm{n}(\%)$ & $93(68)$ \\
\hline Peripheral artery disease, $\mathrm{n}(\%)$ & $4(3)$ \\
\hline Chronic obstructive pulmonary disease, $\mathrm{n}(\%)$ & $5(3.6)$ \\
\hline Heart failure, $\mathrm{n}(\%)$ & $13(9.5)$ \\
\hline Chronic kidney disease, n (\%) & $11(8.0)$ \\
\hline Hypertension, n (\%) & $62(45.3)$ \\
\hline Diabetes, n (\%) & $23(16.8)$ \\
\hline Non-loop diuretics, n (\%) & $38(27.7)$ \\
\hline Loop diuretics, n (\%) & $22(16.1)$ \\
\hline Beta blockers, n (\%) & $61(44.5)$ \\
\hline Calcium channel blockers, $\mathrm{n}(\%)$ & $29(21.2)$ \\
\hline RAS-inhibitors, n (\%) & $50(36.5)$ \\
\hline Acetyl salicylic acid, n (\%) & $76(55.5)$ \\
\hline NSAID, n (\%) & $19(13.9)$ \\
\hline ADP-inhibitors, n (\%) & $38(27.7)$ \\
\hline Statin use, n (\%) & $137(100.0)$ \\
\hline \multicolumn{2}{|l|}{ Dosage of statins (\%) } \\
\hline $20 \mathrm{mg}$ & $13(9.5)$ \\
\hline $40 \mathrm{mg}$ & $64(46.7)$ \\
\hline $80 \mathrm{mg}$ & $60(43.8)$ \\
\hline \multicolumn{2}{|l|}{ Type of statin, n (\%) } \\
\hline Atorvastatin & $72(52.6)$ \\
\hline Fluvastatin & $4(2.9)$ \\
\hline Pravastatin & $5(3.6)$ \\
\hline Rosuvastatin & $7(5.1)$ \\
\hline Simvastatin & $49(35.8)$ \\
\hline Fibrates, n (\%) & $30(21.9)$ \\
\hline Bile acid sequestrant, $\mathrm{n}(\%)$ & $62(45.3)$ \\
\hline Nicotine acid, n (\%) & 39 (28.5) \\
\hline Ezetimibe, n (\%) & $130(94.9)$ \\
\hline $\mathrm{PCl}, \mathrm{n}(\%)$ & $70(51.1)$ \\
\hline CABG, n (\%) & $34(24.8)$ \\
\hline
\end{tabular}

ADP-inhibitors, adenosine diphosphate inhibitors; CABG, coronary artery bypass graft; NSAID, non-steroidal antiinflammatory drugs; $\mathrm{PCl}$, percutaneous coronary intervention; PCSK9 inhibitors, proprotein convertase subtilisin-kexin type 9 inhibitors; RAS-inhibitors, renin-angiotensin system inhibitors.

PCSK9i initiation. No other statistical difference regarding demographics, diseases, concurrent medication or coronary procedures was found between the patients receiving triple therapy compared with the patients not receiving triple LLT (table 2). 


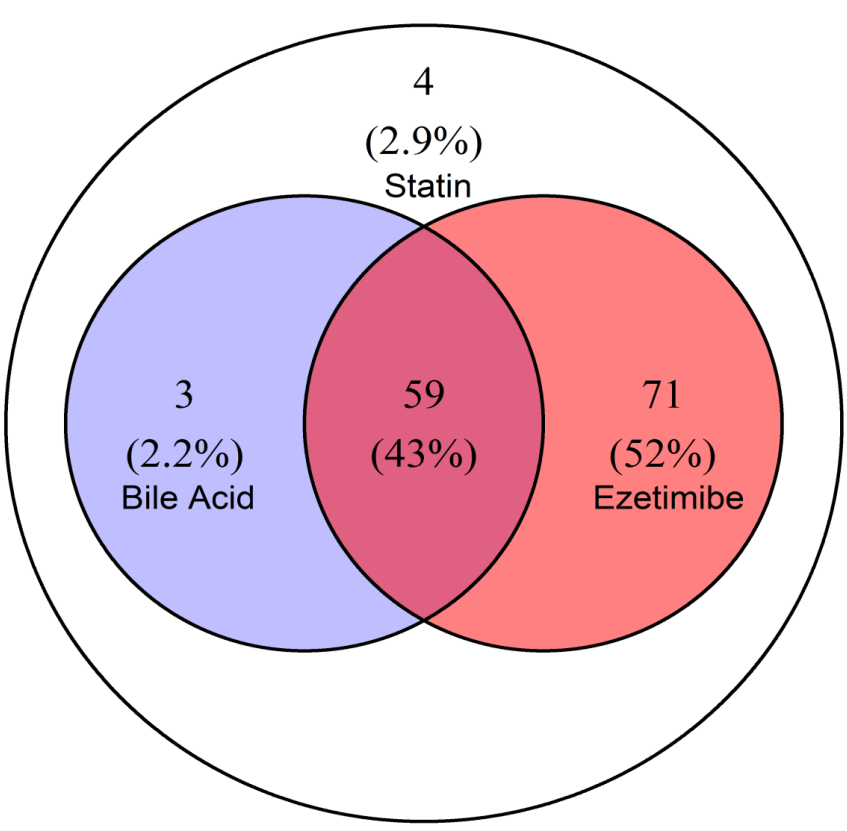

Figure 2 Venn diagram illustrating combinations of prescribed lipid lowering treatment in 137 patients initiated on PCSK9 inhibitors.

\section{DISCUSSION}

In this nationwide study, we described the prevalence and clinical and demographical characteristics of patients treated with PCSK9i over a 1-year period from 2016 to 2017. PCSK9i were prescribed in a total of 137 patients with an increase from 11 initiated patients in the Q1 of 2016 to 40 initiated patients in the Q1 of 2017. Patients were characterised by having a history of IHD and having received PCI and CABG. PCSK9i initiation was consistently clustered in patients attempted to reach maximum tolerated LLT resembled by various types and dosages of statins and patients having attempted dual LLT and half having attempted triple LLT. Patients in dual LLT were more prone to have heart failure than patients attempted in triple LLT prior to PCSK9i initiation.

We found three similar studies describing the clinical characteristics and LLT prior to PCSK9i initiation. ${ }^{16-18}$ In Rane $e t a l$ and Mues $e t a l$, only $54 \%$ and $19 \%$ of patients had received two types of LLT, $24 \%$ and $26 \%$ of patients did not receive any LLT prior to initiation with PCSK9i, respectively and only $17 \%$ of patients had previously received three types of LLT. Also, in Fairman et al and Rane $e t a l, 60 \%$ and $69 \%$ of patients, respectively, were in treatment with statin as monotherapy. The results from these studies are in discrepancy with the present study where $100 \%$ of patients had received statin treatment, $97 \%$ had received minimum two types of LLT and only $3 \%$ were in statin treatment alone. Physician reported intolerability to statin in $32 \%$ of the patients initiated on PCSK9i greatly influenced PCSK9i initiation in Rane et al. However, ezetimibe was attempted surprisingly infrequent (7.6\% of patients) in contrast to the present study where ezetimibe was prescribed in $94.9 \%$ of patients. Differences of eligibility of PCSK9i in the ESC guidelines compared
Table 2 Clinical characteristics, demographics and medication use by number of lipid lowering drugs prescribed (not triple therapy vs triple therapy) prior to initiation of PCSK9 inhibitors

\begin{tabular}{|c|c|c|}
\hline Parameter & $\begin{array}{l}\text { Not triple } \\
\text { treatment }\end{array}$ & $\begin{array}{l}\text { Triple } \\
\text { treatment }\end{array}$ \\
\hline Number of patients, $n$ & 78 & 59 \\
\hline Age, median (IQR) & $61(55-68)$ & $62(56-69)$ \\
\hline Men, n (\%) & $42(53.8)$ & $31(52.5)$ \\
\hline Ischaemic stroke, n (\%) & $6(7.7)$ & $4(6.8)$ \\
\hline Ischaemic heart disease, n (\%) & $54(69.2)$ & $39(66.1)$ \\
\hline $\begin{array}{l}\text { Chronic obstructive pulmonary } \\
\text { disease, } \mathrm{n}(\%)\end{array}$ & $4(5.1)$ & $<3(<5 \%)$ \\
\hline Heart failure, n (\%) & $10(12.8)$ & $3(5.1)$ \\
\hline Chronic kidney disease, n (\%) & $6(7.7)$ & $5(8.5)$ \\
\hline Hypertension, n (\%) & $34(43.6)$ & $28(47.5)$ \\
\hline Diabetes, n (\%) & $14(17.9)$ & 9 (15.3) \\
\hline Non-loop diuretics, n (\%) & $20(25.6)$ & $18(30.5)$ \\
\hline Loop diuretics, n (\%) & $16(20.5)$ & $6(10.2)$ \\
\hline Beta blockers, n (\%) & $30(38.5)$ & $31(52.5)$ \\
\hline Calcium channel blockers, n (\%) & $17(21.8)$ & $15(25.4)$ \\
\hline RAS-inhibitors, n (\%) & $28(35.9)$ & $22(37.3)$ \\
\hline Acetyl salicylic acid, n (\%) & $48(61.5)$ & $28(47.5)$ \\
\hline NSAID, n (\%) & $12(15.4)$ & 7 (11.9) \\
\hline ADP-inhibitors, n (\%) & $17(21.8)$ & $21(35.6)$ \\
\hline \multicolumn{3}{|l|}{ Dosage of statins (\%) } \\
\hline $20 \mathrm{mg}$ & $6(7.7)$ & $7(11.9)$ \\
\hline $40 \mathrm{mg}$ & $40(51.3)$ & $24(40.7)$ \\
\hline $80 \mathrm{mg}$ & $32(41.0)$ & $28(47.5)$ \\
\hline \multicolumn{3}{|l|}{ Type of statin, n (\%) } \\
\hline Atorvastatin & $46(59.0)$ & 33 (55.9) \\
\hline Fluvastatin & $<3(<4 \%)$ & $<3(<5 \%)$ \\
\hline Pravastatin & $<3(<4 \%)$ & $4(6.8)$ \\
\hline Rosuvastatin & $5(6.4)$ & $<3(<5 \%)$ \\
\hline Simvastatin & $26(33.3)$ & 18 (30.5) \\
\hline Fibrates, n (\%) & $14(17.9)$ & $16(27.1)$ \\
\hline Nicotine acid, n (\%) & $20(25.6)$ & 19 (32.2) \\
\hline PCl, n (\%) & $38(48.7)$ & $32(54.2)$ \\
\hline CABG, n (\%) & $22(28.2)$ & $12(20.3)$ \\
\hline
\end{tabular}

ADP, adenosine diphosphate; CABG, coronary artery bypass graft; NSAID, non-steroidal anti-inflammatory drugs; $\mathrm{PCl}$, percutaneous coronary intervention; RAS, renin-angiotensin system.

with the ACC guidelines could possibly also have influenced treatment regimens with LLT prior to PCSK9i initiation. ${ }^{12}{ }^{19-21}$ We observed a more rigorous adherence in attempting statin treatment and additional conventional non-statin treatment prior to initiation of PCSK9i in the present study compared with similar studies and studies of patients at high risk of CVD not receiving PCSK9i. ${ }^{22-24}$ 
The infrequent use of rosuvastatin was primarily due to a protecting marketing patent, making rosuvastatin more expensive than atorvastatin making atorvastatin the first choice of potent statin in Denmark. Simvastatin is primarily used in the process of overcoming side effects of potent statin treatment in patients with IHD in order to reach maximum tolerated dose of LLT or as primary prophylaxis treatment of CVD. In our cohort, 36\% of patients received simvastatin, which most likely indicates attempts of titrating to maximum tolerated dosage of statin when considering the strict national criteria for medicine subsidy regarding PCSK9i treatment and most patients had IHD. This could indicate persistent attempts of reaching LDL-C target goals in adherence to national clinical guidelines, and a last resort usage of PCSK9i.

We found that patients in dual LLT were more prone to have heart failure than patients attempted in triple LLT prior to PCSK9i initiation. The treating physician could possibly be susceptible to more intensive lowering of LDL-C to reduce the risk of future cardiovascular events in the more frail patients such as patients with verified heart failure. Although patients were initiated according to both national and international guidelines, perhaps certain other clinical traits might influence both physicians and committee's approving of medicine subsidy for PCSK9i. ${ }^{25}$ Patients' demographics and concurrent medication use did notsignificantly differ in patients having received triple LLT and patients not having received triple LLT.

Despite having been endorsed by guidelines for patients with many different kinds of atherosclerotic CVD, 66\% of the patients initiated on PCSK9i had a history of IHD (online supplementary appendix figure 2). The large randomised trials investigating the effects of intensified LLT $^{7} 910$ 26-29 or PCSK9i treatment ${ }^{1130-37}$ on reducing future cardiovascular events have predominately included patients with established IHD or a recent IHD related event. The Danish national guidelines have also adapted more accommodative criteria for PCKS9i initiation in patients with a history of IHD, which explains the clustering of PCSK9i in such patients. Repeating our research in another country might show different results regarding the clinical patient characteristics, which probably will become more streamlined along with the publication of long-term randomised trials evaluating PCSK9i treatment.

Even though we observed an increasing number of patients initiated on PCSK9i, only 137 patients in total were prescribed with PCSK9i over the study period. Statins are the first-line treatment, more frequently used and have established effectiveness on improving long-term cardiovascular outcomes in both primary and secondary prevention trials without being extensively expensive compared with PCSK9i.

PCSK9i are without established long-term effectiveness on major cardiovascular events and clinical trials are still ongoing. Based on the current evidence of the effectiveness of PCSK9i on reducing major cardiovascular events, the Institute for Clinical and Economic Review (ICER) reported a number needed to treat for 5 years to avoid one major cardiovascular event (NNT5) of 28 individuals. ${ }^{38}$ While randomised clinical trials of PCSK9i have shown beneficial efficacy, they are very expensive and this may have had major influence on theoverall clinical implementation. The cost ofPCSK9i is around $\$ 14350$ per year in 2017, generating a cost-effectiveness ratio which may exceed a threshold of price pr. quality-affected life years. These economic considerations together with a possible postponed apprising of PCSK9i as a treatment option may explain why PCSK9i have been used rather infrequent when also taking into account how many patients who actually need further LLT. ${ }^{39} 40$ Economic considerations can also help explain the modified and individually formed national guidelines for PCSK9i initiation. Committing fully to the criteria for PCSK9i initiation stated in the ESC or ACC guidelines would probably not be realistic for any countries' medical expenditures, resulting in many patients being rejected for medicine subsidy and patients with certain clinical characteristics being favored ${ }^{25}$. Ongoing trials will help establish whether further lowering of LDL-C has sufficient beneficial effects to implement PCSK9i on a wider scale and whether the cost-effectiveness of such implementation is favourable. In conclusion, conventional LLT had its core role in a first line of treatment to provide an initial assessment of LDL-C lowering by using various types of statin in various dosages with frequent attempts of double and triple LLT prior to PCSK9i initiation in patients in high or very high risk of CVD. The main usage of PCSK9i were in patients predominately with IHD in treatment with statin and ezetemibe in agreement with current national and international clinical guidelines.

\section{Strengths and limitations of this study}

In the present study, we utilised the Danish national registries to perform a description of the characteristics of patients prescribed with PCSK9i on a national basis. We were able to include an unselected cohort of patients, with a great variety of comorbidities. The Danish nationwide registries include data on all residents regardless of participation in the labour market and therefore are not affected by selection bias caused by including selected age groups, hospitals or health insurance systems.

Patients with familial hypercholesterolaemia (FH), who are also eligible for PCSK9i treatment, collect their PCSK9i at a specialised hospital unit and due to the design of the present study, we were not able to identify patients with a diagnosis of $\mathrm{FH}$.

Due to the observational design of the present study, we were not able to retrieve data regarding biochemical analyses, clinical reasons for initiation of PCSK9i, LLT after initiation of PCSK9i or adverse events with statin treatment. Both statin and non-statin treatment in different dosages were applied in almost every patient in the present study, which may reflect clinical decisions of resolving intolerable adverse effects, reaching LDL-C target levels 
or strict adherence to guidelines. Due to a low diagnostic sensibility, we were not able to fully discriminate the indications of patients receiving PCSK9i.

We did not include a comparative cohort of patients not receiving PCSK9i; however, we were able to compare clinical characteristics, comorbidities and demographics in the present study with an observational study investigating CVD and LLT in the Danish general population. ${ }^{39}$

\section{Author affiliations}

${ }^{1}$ Department of Cardiology, Rigshospitalet, Copenhagen, Denmark

${ }^{2}$ Department of Cardiology, Copenhagen University Hospital, Bispebjerg, Copenhagen, Denmark

${ }^{3}$ Department of Cardiology, Copenhagen University Hospital Herlev and Gentofte, Hellerup, Denmark

${ }^{4}$ Novo Nordisk Søborg, Novo Nordisk AS, Bagsvaerd, Denmark

Contributors JSJ: Contributions to the design, analysis and interpretation of data for the work. Drafted the manuscript. Final approval for version to be published. PEW: Conception and design, interpretation and analysis of data, revising of manuscript, final approval for publishing. LEB: Interpretation and analysis of data, revising of manuscript, final approval for publishing. DEH: Interpretation and analysis of data, revising of manuscript, final approval for publishing. MSR Conception and design, revising of manuscript, final approval for publishing. A-MS: Conception and design, revising of manuscript, final approval for publishing. JET: Conception and design, revising of manuscript, final approval for publishing. LVK: Interpretation and analysis of data, revising of manuscript, final approval for publishing. GHG: Conception and design, revising of manuscript, final approval for publishing. JP: Acquisition of data, conception and design, interpretation and analysis of data, revising of manuscript, final approval for publishing.

Funding The authors have not declared a specific grant for this research from any funding agency in the public, commercial or not-for-profit sectors.

Competing interests None declared.

Patient consent for publication Not required.

Ethics approval The Danish Data Protection Agency approved this study (ref. no.: 2007-58-0015/GEH- 2014 - 016 I-Suite no.: 02734)

Provenance and peer review Not commissioned; externally peer reviewed.

Open access This is an open access article distributed in accordance with the Creative Commons Attribution Non Commercial (CC BY-NC 4.0) license, which permits others to distribute, remix, adapt, build upon this work non-commercially, and license their derivative works on different terms, provided the original work is properly cited, appropriate credit is given, any changes made indicated, and the use is non-commercial. See: http://creativecommons.org/licenses/by-nc/4.0/.

\section{REFERENCES}

1. Verschuren WM, Jacobs DR, Bloemberg BP, et al. Serum total cholesterol and long-term coronary heart disease mortality in different cultures. Twenty-five-year follow-up of the seven countries study. JAMA 1995;274:131-6 http://www.ncbi.nlm.nih.gov/pubmed/ 7596000

2. Castelli WP, Anderson K, Wilson PW, et al. Lipids and risk of coronary heart disease. The Framingham Study. Ann Epidemiol;2:23-8 http:// www.ncbi.nlm.nih.gov/pubmed/1342260

3. Ference BA, Ginsberg HN, Graham I, et al. Low-density lipoproteins cause atherosclerotic cardiovascular disease. 1. Evidence from genetic, epidemiologic, and clinical studies. A consensus statement from the European Atherosclerosis Society Consensus Panel. Eur Heart J 2017;38:2459-72.

4. Mihaylova B, Emberson J, Blackwell L, et al. The effects of lowering LDL cholesterol with statin therapy in people at low risk of vascular disease: meta-analysis of individual data from 27 randomised trials. Lancet 2012;380:581-90.

5. Thavendiranathan P, Bagai A, Brookhart MA, et al. Primary Prevention of Cardiovascular Diseases With Statin Therapy. Arch Intern Med 2006;166:2307.

6. Heart Protection Study Collaborative Group. MRC/BHF Heart Protection Study of cholesterol lowering with simvastatin in 20,536 high-risk individuals: a randomised placebo-controlled trial. Lancet 2002;360:7-22.

7. Cannon $\mathrm{CP}$, Braunwald E, McCabe $\mathrm{CH}$, et al. Intensive versus moderate lipid lowering with statins after acute coronary syndromes. N Engl J Med 2004;350:1495-504.

8. Stitziel $\mathrm{NO}$, Won $\mathrm{HH}$, Morrison AC, et al. Inactivating mutations in NPC1L1 and protection from coronary heart disease. N Engl J Med 2014;371:2072-82.

9. Cannon CP, Blazing MA, Giugliano RP, et al. Ezetimibe Added to Statin Therapy after Acute Coronary Syndromes. N Engl J Med 2015;372:2387-97

10. Baigent $\mathrm{C}$, Blackwell L, Emberson J, et al. Efficacy and safety of more intensive lowering of LDL cholesterol: a meta-analysis of data from 170,000 participants in 26 randomised trials. Lancet 2010;376:1670-81.

11. Sabatine MS, Giugliano RP, Wiviott SD, et al. Efficacy and safety of evolocumab in reducing lipids and cardiovascular events. $N$ Engl J Med 2015;372:1500-9.

12. Landmesser U, John Chapman M, Farnier M, et al. European Society of Cardiology/European atherosclerosis society task force consensus statement on proprotein convertase subtilisin/kexin type 9 inhibitors: practical guidance for use in patients at very high cardiovascular risk. Eur Heart J 2016;14:ehw480.

13. Lloyd-Jones DM, Morris PB, Ballantyne CM, et al. ACC expert consensus decision pathway on the role of non-statin therapies for LDL-cholesterol lowering in the management of atherosclerotic cardiovascular disease risk. J Am Coll Cardiol 2016;2016:92-125.

14. Kernan WN, Ovbiagele B, Black HR, et al. Guidelines for the prevention of stroke in patients with stroke and transient ischemic attack: a guideline for healthcare professionals from the American Heart Association/American Stroke Association. Stroke 2014;45:2160-236.

15. Gerhard-Herman MD, Gornik HL, Barrett C, et al. 2016 AHA ACC guideline on the management of patients with lower extremity peripheral artery disease: executive summary. Vasc Med 2017;22:1465-508.

16. Rane PB, Patel J, Harrison DJ, et al. Patient characteristics and realworld treatment patterns among early users of PCSK9 Inhibitors. Am J Cardiovasc Drugs 2018;18:103-8.

17. Mues K, Bratton E, Wirtz HS, et al. Abstract 19090: patient characteristics and lipid lowering treatment of early adopters of PCSK9i therapies in the United States. Circulation 2017;136.

18. Fairman KA, Davis LE, Sclar DA. Real-world use of PCSK-9 inhibitors by early adopters: cardiovascular risk factors, statin co-treatment, and short-term adherence in routine clinical practice. Ther Clin Risk Manag 2017;13:957-65.

19. Lloyd-Jones DM, Morris PB, Ballantyne CM, et al. 2017 Focused Update of the 2016 ACC expert consensus decision pathway on the role of non-statin therapies for LDL-cholesterol lowering in the management of atherosclerotic cardiovascular disease risk. J Am Coll Cardiol 2017;70:1785-822.

20. Sabatine MS. Proprotein convertase subtilisin/kexin type 9 (PCSK9) inhibitors: comparing and contrasting guidance across the Atlantic. Eur Heart J 2017;38:ehw572.

21. Gencer B, Koskinas KC, Räber L, et al. Eligibility for PCSK9 Inhibitors According to American College of Cardiology (ACC) and European Society of Cardiology/European Atherosclerosis Society (ESC/EAS) Guidelines After Acute Coronary Syndromes. J Am Heart Assoc 2017;6:e006537.

22. Kuiper JG, Sanchez RJ, Houben E, et al. Use of Lipid-modifying Therapy and LDL-C Goal attainment in a high-cardiovascular-risk population in the Netherlands. Clin Ther 2017;39:819-27.

23. Davidson MH, Maki KC, Pearson TA, et al. Results of the National Cholesterol Education (NCEP) Program Evaluation ProjecT Utilizing Novel E-Technology (NEPTUNE) II survey and implications for treatment under the recent NCEP Writing Group recommendations. Am J Cardiol 2005;96:556-63.

24. Karalis DG, Subramanya RD, Hessen SE, et al. Achieving optimal lipid goals in patients with coronary artery disease. Am J Cardiol 2011;107:886-90.

25. Hess GP, Natarajan P, Faridi KF, et al. Proprotein convertase subtilisin/kexin type 9 inhibitor therapy. Circulation 2017;136:2210-9.

26. LaRosa JC, Grundy SM, Waters DD, et al. Intensive lipid lowering with atorvastatin in patients with stable coronary disease. $N$ Engl J Med 2005;352:1425-35.

27. Pedersen TR, Faergeman O, Kastelein JJ, et al. High-dose atorvastatin vs usual-dose simvastatin for secondary prevention after myocardial infarction: the IDEAL study: a randomized controlled trial. JAMA 2005;294:2437.

28. Armitage J, Bowman L, Wallendszus $\mathrm{K}$, et al. Intensive lowering of LDL cholesterol with 80 mg versus 20 mg simvastatin daily in 12,064 
survivors of myocardial infarction: a double-blind randomised trial. Lancet 2010;376:1658-69.

29. de Lemos JA, Blazing MA, Wiviott SD, et al. Early intensive vs a delayed conservative simvastatin strategy in patients with acute coronary syndromes: phase $\mathrm{Z}$ of the $\mathrm{A}$ to $\mathrm{Z}$ trial. JAMA 2004;292:1307.

30. Tice JA, Tingley W, Mosesova S, et al. Effects of RG7652, a monoclonal antibody against proprotein convertase subtilisin/kexin type 9, on LDL cholesterol in patients with coronary heart disease or high risk: results from.

31. Blom DJ, Hala T, Bolognese M, et al. A 52-week placebocontrolled trial of evolocumab in hyperlipidemia. N Engl J Med 2014;370:1809-19.

32. Sabatine MS, Giugliano RP, Keech AC, et al. Evolocumab and clinical outcomes in patients with cardiovascular disease. N Engl J Med 2017:NEJMoa1615664.

33. Kereiakes DJ, Robinson JG, Cannon CP, et al. Efficacy and safety of the proprotein convertase subtilisin/kexin type 9 inhibitor alirocumab among high cardiovascular risk patients on maximally tolerated statin therapy: the odyssey combo i study. Am Heart $J$ 2015;169:906-15.

34. Cannon CP, Cariou B, Blom D, et al. Efficacy and safety of alirocumab in high cardiovascular risk patients with inadequately controlled hypercholesterolaemia on maximally tolerated doses of statins: the odyssey combo ii randomized controlled trial. Eur Heart $J$ 2015;36:1186-94.

35. Robinson JG, Farnier M, Krempf M, et al. Efficacy and safety of alirocumab in reducing lipids and cardiovascular events. $N$ Engl $J$ Med 2015;372:1489-99.

36. Bays $\mathrm{H}$, Gaudet D, Weiss $\mathrm{R}$, et al. Alirocumab as add-on to atorvastatin versus other lipid treatment strategies: odyssey options i randomized trial. J Clin Endocrinol Metab 2015;100:3140-8.

37. Farnier M, Jones P, Severance R, et al. Efficacy and safety of adding alirocumab to rosuvastatin versus adding ezetimibe or doubling the rosuvastatin dose in high cardiovascular-risk patients: The odyssey options ii randomized trial. Atherosclerosis 2016;244:138-46.

38. Tice JA, Ollendorf DA, Chapman R, et al. Institute for Clinical and Economic Review. Evolocumab for Treatment of High Cholesterol: Effectiveness and Value New Evidence Update. 2017. https://icerreview.org/material/pcks9-inhibitors-neu/.

39. Siggaard-Andersen N, Freiberg JJ, Nordestgaard BG. Only a fraction of patients with ischaemic diseases or diabetes are treated to recommended target values for plasma lipids. Dan Med $J$ 2012;59:A4470.

40. Benn M, Watts GF, Tybjaerg-Hansen A, et al. Familial hypercholesterolemia in the Danish general population: prevalence, coronary artery disease, and cholesterol-lowering medication. J Clin Endocrinol Metab 2012;97:3956-64. 